Recherches en didactique des langues et des cultures

Les cahiers de l'Acedle

11-2| 2014

Plurilinguisme(s) et entreprise : enjeux didactiques

Combiner didactique des langues, psycholinguistique et psychologie cognitive pour créer un outil pédagogique de lecture en français langue seconde

\title{
Géraldine Charles-Dominique
}

\section{OpenEdition}

1 Journals

Édition électronique

URL : http://journals.openedition.org/rdlc/1819

DOI : $10.4000 /$ rdlc. 1819

ISSN : 1958-5772

Éditeur

ACEDLE

Référence électronique

Géraldine Charles-Dominique, «Combiner didactique des langues, psycholinguistique et psychologie cognitive pour créer un outil pédagogique de lecture en français langue seconde », Recherches en didactique des langues et des cultures [En ligne], 11-2 | 2014, mis en ligne le 07 juin 2014, consulté le 30 avril 2019. URL : http://journals.openedition.org/rdlc/1819 ; DOI : 10.4000/rdlc.1819

Ce document a été généré automatiquement le 30 avril 2019

\section{c) (7) $९$}

Recherches en didactique des langues et des cultures is licensed under a Creative Commons AttributionNonCommercial-NoDerivatives 4.0 International License 


\title{
Combiner didactique des langues, psycholinguistique et psychologie cognitive pour créer un outil pédagogique de lecture en français langue seconde
}

\author{
Géraldine Charles-Dominique
}

\section{Présentation de l'étude}

1 Nombreux sont les étudiants qui suivent un cursus à l'étranger, en langue seconde. Le niveau d'exigence qui leur est imposé est généralement le même que pour les étudiants natifs. Il leur est ainsi couramment demandé de lire plusieurs documents pour faire un exposé, un compte-rendu écrit ou pour en discuter à l'oral de manière pertinente. Or, comprendre globalement ce que dit chaque texte ne peut suffire puisqu'à un niveau universitaire, il faut pouvoir comprendre de manière approfondie le contenu et la source des documents, en extraire les informations pertinentes, et en faire une lecture transversale et critique. Nous verrons d'une part, que lire plusieurs documents est une tâche particulièrement exigeante, et d'autre part, que la lecture en langue seconde est moins efficace qu'en langue première, même chez des lecteurs de niveau avancé (Gaonac'h, 2005). Une intervention pédagogique serait alors nécessaire pour soutenir les efforts du lecteur qui se trouve face à plusieurs textes en langue seconde.

Une étude a donc été menée en contexte universitaire auprès d'étudiants en français langue seconde dans le but de tester un outil pédagogique ${ }^{1}$ conçu pour optimiser leur compréhension de l'écrit.

3 L'outil qui a été élaboré pour cette étude s'inspire de recherches théoriques issues de la didactique des langues, de la psycholinguistique et de la psychologie cognitive (cadre 
théorique décrit plus loin). Sur le plan expérimental, nous avons analysé les données et mesuré l'impact de l'outil grâce à certaines méthodes pratiquées en psycholinguistique et en psychologie cognitive. Notre guidage, utilisé par une partie des participants, semble les avoir aidés à approfondir leur travail de compréhension.

\section{Lire dans un contexte exigeant}

4 Cette étude a plongé des apprenants de français langue seconde dans un contexte exigeant de lecture non seulement parce que la lecture en langue seconde implique une charge cognitive supérieure (comparé à la lecture en langue première), mais également parce qu'ils ont aussi dû lire et exploiter plusieurs textes sur un thème controversé.

5 Sur le plan cognitif, face à un seul texte, la représentation de la source ${ }^{2}$ est associée à celle $\mathrm{du}$ texte. La représentation de textes uniques repose donc sur un modèle simple: une source - un contenu. Par contre, la représentation d'un ensemble de documents demande au lecteur de se construire un modèle de situation plus complexe. Selon Rouet (2006), cette représentation multidocumentaire comprendrait trois types de liens représentationnels : les liens entre les contenus des textes, les liens entre les sources des textes, et les liens entre chaque contenu textuel et sa source. Par conséquent, le fait de comprendre qui dit quoi ? est salutaire pour le lecteur puisque les liens entre sources et contenus servent de piliers dans la compréhension multidocumentaire. En didactique des langues, Garcia-Debanc (1989) a quant à elle cherché, par la pratique guidée du tri de texte, à développer chez de jeunes lecteurs la capacité à établir des liens ou des différences entre plusieurs textes : repérer rapidement le type de texte ferait donc partie des habiletés à renforcer chez les lecteurs.

6 Par ailleurs, l'identification de la source joue un rôle clé dès lors que le lecteur fait face à plusieurs textes: quand les sources sont mieux comprises (d'où vient l'information lue dans tel texte?) une amélioration est visible au niveau de la compréhension inter- et intratextuelle (c'est-à-dire la compréhension des relations entre les textes et la compréhension de chaque texte) (Strømsø, Bråten \& Britt, 2009). Rouet (2006; 2011), Strømsø, Bråten, et Britt (2009), à travers leurs travaux convergents en psychologie de la lecture, considèrent ainsi les sources comme un appui à la compréhension et à l'intégration du contenu.

7 De plus, savoir émettre des hypothèses sur le type de document rencontré (plus ou moins expert dans le domaine abordé, plus ou moins crédible) serait une compétence qui favorise la compréhension des textes et leur bonne exploitation. Par exemple, une étude de Limón et Vidal (2011), menée sur 100 étudiants espagnols, a montré que ceux qui ont un plus haut niveau de compétence épistémique passent plus de temps à étudier les documents à lire, pour en retirer un plus grand bénéfice (plus d'informations apprises dans les documents).

8 En langue seconde, une étude sur la lecture multidocumentaire de Gebril et Plakans (2009) vient renforcer l'idée d'un lien entre compréhension du contenu et compréhension des sources. Dans une tâche intégrée de lecture-écriture en anglais langue seconde, les étudiants qui ont eu plus de mal à comprendre les textes ont moins cité les sources que les autres, dans leurs rédactions basées sur les textes lus.

C'est pourquoi, dans notre étude, nous avons voulu attirer l'attention du lecteur sur les sources des documents de façon à ce qu'il distingue mieux les textes entre eux, mais aussi 
pour qu'il aiguise son esprit critique quant à la crédibilité des textes. La prise en compte des sources par le lecteur (références aux sources dans une synthèse de documents) a par ailleurs été considérée comme un indicateur de compréhension.

Enfin, nous pourrions ajouter que chercher de l'information dans des textes est une tâche complexe lorsqu'il s'agit de répondre à des questions qui demandent de revoir et d'intégrer plusieurs éléments du texte, sur de larges parties; ces questions sont dites de haut niveau car elles sollicitent chez le lecteur les processus cognitifs de haut niveau (processus situés au-delà du déchiffrage du texte ou de la localisation d'une information dans le texte) et nécessitent une plus grande attention du lecteur (Rouet, Vidal-Abarca, Bert-Erboul \& Millogo, 2001).

11 De même, sélectionner dans les textes certaines informations pour les réutiliser dans une tâche de production écrite demande des efforts cognitifs soutenus : dans une tâche de lecture-écriture, les opérations mentales du lecteur passent ainsi par la construction d'un modèle de tâche (représentation mentale des opérations à mettre en exécution tout au long de la tâche), l'évaluation des informations nécessaires pour exécuter la tâche, la sélection des informations (dans les consignes et dans les textes), l'activation et l'intégration des informations, l'exécution de la tâche, et l'évaluation de la qualité de sa production écrite ; précisons aussi que de nouvelles planifications peuvent avoir lieu en cours de tâche ainsi que des contrôles réguliers de ce qui a déjà été fait ou doit être fait (Rouet \& Britt, 2011).

Bien que travailler sur plusieurs textes relève du quotidien de tout étudiant, nous devons donc être conscients de la complexité impliquée au niveau cognitif. Le risque, face à plusieurs textes, est de ne plus savoir qui dit quoi, de comprendre les idées générales des textes sans avoir pu pousser le travail de compréhension dans le détail, ou encore, de lire les documents sans s'interroger sur leur crédibilité. Le lecteur doit être capable de sélectionner les informations pertinentes dans les documents, de les relier entre elles quand cela se révèle pertinent, et de gérer sa tâche de lecture de manière stratégique et méthodique (bonne gestion du temps, retour sur les passages mal compris...). Nous avons donc proposé aux lecteurs un outil qui puisse les guider pour mieux comprendre et exploiter les textes à lire.

\section{Comprendre des textes en langue seconde}

Concentrons-nous à présent sur la spécificité de la lecture en langue seconde. Lire en langue seconde impliquerait des processus de bas niveau moins efficaces (Bernhardt, 1987) car la reconnaissance des structures syntaxiques et des mots (parfois inconnus) est plus lente. Les stratégies de compréhension en langue seconde sont souvent les mêmes que celles utilisées en langue première (ou L1), mais elles sont souvent moins efficaces et systématiques qu'en L1 (Block, 1986). Or, un déchiffrage peu efficace pourrait empêcher le lecteur de prendre de la hauteur de vue et de comprendre globalement la situation décrite (Acuňa, 2005). Cela peut poser problème face à des textes en langue seconde :

[...] l'efficacité de la reconnaissance des mots (ce que l'on appelle l'accès lexical), c'est-à-dire pour l'essentiel la vitesse de cette reconnaissance, constitue une condition nécessaire pour que des processus de plus haut niveau puissent être mis en ceuvre par le lecteur (Gaonac'h, $2005: 1$.

14 Certes, le niveau d'automaticité des processus de bas niveau (relatifs au déchiffrage) est plus élevé chez les apprenants de niveau avancé que chez ceux de niveau débutant ou 
intermédiaire, mais il freine malgré tous les processus de haut niveau (accès au sens des phrases, planification d'une tâche de lecture...), et la planification de la tâche s'en trouverait plus difficile à mettre en œuvre (Coirier, Gaonac'h, \& Passerault, 1996).

Bien sûr, l'apprenant qui lit en langue seconde n'est pas dénué d'atouts : il peut s'appuyer sur des connaissances ou des savoir-faire issus de sa langue première pour comprendre les textes (Meissner et al., 2004) ainsi que sur ses capacités cognitives (rétention temporaire des informations) habituellement sollicitées dans la lecture en langue première (Payne, Kalibatseva \& Jungers, 2009) ; mais cela ne va pas de soi puisque des faiblesses linguistiques peuvent gêner ces transferts bénéfiques à la compréhension (Coirier, Gaonac'h, \& Passerault, 1996), tout comme le niveau d'automaticité des processus de bas niveau que nous avons déjà abordé.

Face à plusieurs textes, il se pourrait qu'un lecteur de langue seconde ait un effort trop important à fournir, et qu'il soit noyé dans l'information. Mais la compréhension multidocumentaire peut être exercée en classe de langue : une étude menée auprès de lecteurs en français langue seconde (Smuk, 2011) a ainsi montré qu'un entraînement à la synthèse a fini par améliorer la compréhension des documents ainsi que la capacité des étudiants à les décrire.

Enfin, nous pouvons noter le rôle clé des stratégies globales qui ont pu être observées en langue seconde chez les bons compreneurs (Plakans, 2009; Huang, Chern, \& Lin, 2009; Kai, 2011). Une stratégie globale consiste à regrouper plusieurs éléments d'un texte sous une idée clé ou une catégorie pour mieux les retenir : il s'agit par exemple de retenir l'idée principale d'un paragraphe et de la noter sur un brouillon. En didactique, ces stratégies ont été mises en avant dans l'approche globale introduite par Moirand et Lehmann dans les années 1970 : il s'agit de guider le lecteur du connu vers l'inconnu, du global vers les détails des textes (Moirand, 1979), ce qui donne au lecteur des points d'appui pour affiner progressivement sa compréhension. Plus récemment, Bondrea (2011) a proposé à des apprenants de français de lire et d'exploiter un article de presse en plusieurs étapes :

1. prélecture globale : repérage du qui, quoi, comment, quand, où? et travail d'anticipation ancré dans la recherche d'indices dans le texte.

2. lecture - recherche allant plus dans le détail des textes.

3. production individuelle consistant à exprimer sa réaction après la lecture.

18 Cette lecture en deux temps (phases 1 et 2) permettrait de dissocier les difficultés de compréhension pour mieux les dépasser : elle favorise d'abord l'intégration des idées générales et l'élaboration d'hypothèses (par l'anticipation du contenu), puis une recherche ciblée des informations détaillées du texte. L'approche globale des textes a aussi été testée avec succès en anglais langue seconde dans une étude de psycholinguistique (Chen \& Graves, 1995). D'une certaine manière, avec l'approche globale, nous nous plaçons dans une logique d'allègement de la charge cognitive, particulièrement problématique dans la lecture en langue seconde, comme nous l'avons déjà remarqué ; cette logique de dissociation des difficultés rejoint par ailleurs le principe de la double lecture : lire un texte à deux reprises permettrait de favoriser les processus de haut niveau (inférences, accès aux concepts...) dans un second temps (Gaonac'h, 2005). L'idée d'une lecture par étape a alors influencé notre démarche pédagogique dans cette étude. 


\section{Principes du guidage et scénario pédagogique proposé}

Résumons donc les grands principes de notre guidage, qui consiste en un soutien méthodologique de la lecture multidocumentaire en langue seconde.

Le guidage est particulièrement empreint de l'approche globale (Moirand, 1979) qui consiste à aller du connu vers l'inconnu, par étapes, pour permettre une meilleure compréhension des textes en langue seconde.

Rappelons le rôle clé des stratégies globales qui ont pu être observées chez les bons compreneurs (Plakans, 2009 ; Huang, Chern, \& Lin, 2009; Kai, 2011), et donc la pertinence de susciter ce type de stratégies chez le lecteur. Par ailleurs, nous gardons à l'esprit les contraintes liées à la lecture en langue seconde (charge cognitive, lecture un peu plus lente qu'en langue première, possibilité de rencontrer des mots inconnus...) et nous soulignons à cet égard l'intérêt du principe de la double lecture (Gaonac'h, 2005) qui consiste à dissocier des difficultés pour favoriser les processus de haut niveau en seconde lecture. Ainsi, nous nous inscrivons dans une logique de lecture par étapes puisque le guidage incite le lecteur à avoir une première lecture de survol des documents avant d'approfondir son travail de compréhension (en exécutant des tâches de compréhension).

2 De plus, lire des textes aux points de vue variés, et chercher de l'information dans ces textes dans le cadre d'une tâche complexe (questions sur les textes, synthèse à rédiger) entraîne des processus mentaux complexes chez le lecteur (Rouet \& Britt, 2011). Le guidage vise donc à favoriser l'intégration de l'architecture multidocumentaire (comprenant les sources et leurs contenus) pour que le lecteur intègre plus efficacement le "qui dit quoi ?" dans son processus de compréhension. La question du "qui ?" a son importance : demander au lecteur des informations sur les sources pourrait l'aider à les différencier entre elles, mais aussi à les aborder sous un angle critique. Le guidage de notre étude interpelle donc le lecteur sur les sources documentaires.

23 Plus précisément, le guidage se présente sous la forme d'une feuille A4 et doit être complété par l'apprenant en début de lecture (cf. Annexe 1). La première partie du guidage (partie A) est centrée sur les sources : une question sur l'expertise des sources (L'auteur connaît-il bien l'écologie ?) puis une autre sur leur crédibilité (Faites-vous confiance à ce document?) sont posées au lecteur, qui doit y répondre par le biais d'une échelle d'évaluation. Cette première partie a été proposée pour que les textes soient tous abordés dès le début de la séance de travail (approche globale multidocumentaire) et pour inciter le lecteur à prendre en compte les sources. Ces deux questions sur les sources se présentent sous forme de tableau à double entrée, ce qui permet au lecteur de le remplir soit par l'angle de chaque texte, soit de façon transversale, par question (il peut ainsi adopter la stratégie qu'il souhaite).

24 La seconde partie du guidage (partie B) est axée sur les idées principales de chaque texte (il faut relier chaque idée au bon texte) : cet exercice vise à faciliter l'intégration des idées-clés en lien avec les bonnes sources. Afin que la tâche ne soit pas trop évidente, deux distracteurs (idées hors textes) ont été insérés, l'apprenant en étant prévenu dans la consigne.

Le guidage cherche donc à :

- favoriser la compréhension des textes, jusque dans les détails ; 
- et à renforcer l'attention portée aux sources, de manière à permettre une meilleure appréciation des sources (crédibilité de chaque texte) et faciliter l'intégration du modèle multidocumentaire de situation (qui dit quoi ?).

Le temps d'utilisation du guidage a été limité à 10 minutes de façon à favoriser, dans la première phase de lecture (lecture avec guidage), la compréhension globale multidocumentaire. Le but recherché ici est d'alléger la charge cognitive du lecteur pour lui permettre ensuite (grâce à des tâches de compréhension) d'aller plus loin dans la compréhension détaillée intra- et intertextuelle.

Prendre des notes en lisant peut déjà faciliter la compréhension (Olive \& Piolat, 2011), mais un guidage spécifique a été proposé ici (plutôt qu'une feuille vierge) pour fonctionner comme une prise de note optimisée. Prendre des notes librement peut notamment présenter un inconvénient lié aux habitudes de la plupart des lecteurs, toutes origines confondues : on observe que, spontanément, ils prennent des notes sur le texte 1 , puis sur le texte 2, et ainsi de suite..., or, il se peut que le lecteur n'ait pas le temps de noter des informations sur le dernier texte (Charles-Dominique, 2012). Ce risque est ici évité car notre guidage conduit le lecteur à prendre en compte tous les textes dès le début de la séance.

Sur le plan didactique, notre démarche rejoint en quelque sorte celle du Français sur Objectif Universitaire (FOU), où l'on cherche à préparer les étudiants aux études universitaires en leur permettant notamment de développer des compétences d'ordre méthodologique (Borau, 2012), sachant que notre guidage est spécifiquement centré sur la méthodologie de la lecture.

Afin d'exploiter les textes à lire et de récolter des données sur la compréhension, deux tâches ont été proposées :

D'abord, les participants ont répondu à un QCM (Questionnaire à Choix Multiple) comportant 48 questions sur les textes (40 sur les contenus, et 8 sur les sources).

Ensuite, les étudiants ont eu un quart d'heure pour rédiger une synthèse sur les textes lus, en français: cette activité s'intègre au contexte de leur formation puisqu'ils étaient dans un cours sur la synthèse de documents en français langue seconde.

Rappelons d'abord que le QCM présente l'intérêt d'être un support peu coûteux sur le plan cognitif (Alptekin \& Erçetin, 2009) puisqu'il reste centré sur la réception et ne demande pas au lecteur d'écrire des phrases pour répondre. Alptekin et Erçetin (2009) ont ainsi remarqué qu'un matériel pédagogique coûteux en efforts cognitifs (question ouverte du type "Qu'avez-vous compris de telle théorie ?") peut entraîner une surcharge cognitive néfaste à la compréhension. Ils conseillent donc aux concepteurs pédagogiques de proposer des outils d'évaluation de la compréhension basés sur la reconnaissance des informations (plutôt que sur le rappel libre) pour accompagner l'effort en mémoire de travail chez l'apprenant qui lit en langue seconde. Par ailleurs, le QCM permet de limiter le biais de la production écrite dans la langue d'apprentissage : en effet, il se peut que la rédaction de l'apprenant reflète mal ce qu'il a vraiment compris, notamment s'il n'a pas utilisé les bonnes expressions dans sa réponse, cela pourrait notamment faire dévier la problématique du bien comprendre vers le bien écrire (qui n'est pas l'objet de cette étude).

31 Afin d'éviter le risque de la réponse au hasard, cinq réponses possibles (au minimum) ont été conçues (sauf pour les questions intertextuelles, qui demandent de préciser si tel et tel textes partagent une opinion), la réponse "je ne sais pas" était aussi proposée (sixième 
réponse possible). Les questions sur les sources demandaient au lecteur d'évaluer la crédibilité et l'objectivité de chaque texte sur une échelle (de 1 à 5) allant de "pas du tout sérieux" à "très sérieux" et de "pas du tout objectif" à "très objectif" (cf. Annexes 3 et 5). Enfin, pour être certain que les questions du QCM soient bien comprises, leur traduction en anglais a parfois été ajoutée puisque cette langue se trouvait être la langue véhiculaire des participants. La mauvaise interprétation d'une question aurait pu, en effet, être analysée comme une mauvaise compréhension d'un passage textuel, raison pour laquelle les questions du QCM ont parfois été clarifiées. Néanmoins, il ne s'agit pas de rejeter les tâches autres que le QCM : l'expérience de Wahl (2001), en didactique des langues, nous invite ainsi à exploiter la complémentarité des méthodes d'évaluation existantes. Après avoir testé auprès de ses apprenants allophones les questions vrai/faux, les QCM, les questions ouvertes, le résumé de texte, la traduction, et les questions basées sur la grammaire (par exemple : « Que remplace "il" ? Réponse : "il" désigne "le régime nazi" »), Wahl conclut que chaque méthode a ses limites et ne peut totalement refléter la compréhension: il propose donc de varier les méthodes (dans une même séance de lecture) pour obtenir divers indices sur le niveau de compréhension du lecteur. Dans notre étude, la production écrite a ainsi complété le QCM en fournissant des données qui reflètent autrement ce qui a été compris dans les textes.

\section{Précisions sur la méthode}

Dans un centre universitaire de français langue étrangère, seize étudiants allophones de toutes origines ont été répartis en deux groupes de niveaux linguistiques équivalents (C1C2), et de profils linguistiques équilibrés (quand deux participants avaient la même langue 1, ils étaient placés dans deux groupes différents). Par ailleurs, nous avons veillé à avoir des groupes homogènes sur le plan de la répartition homme / femme. Cette séance de travail leur a été présentée comme l'occasion d'un entrainement (non noté) à la compréhension et à la synthèse de documents. Ils ont accepté l'exploitation de leurs productions (sous étiquette anonyme) pour la recherche.

Les participants ont lu quatre textes en français sur le thème du réchauffement climatique : la problématique sous-jacente était axée sur la responsabilité de l'homme dans le réchauffement climatique. Les quatre textes (cf. Annexe 2) respectent la structure suivante :

- deux textes sont de type expert,

- deux textes ne sont pas de type expert.

Les avis exprimés dans les textes sont symétriquement répartis entre le pour et le contre : dans les textes de type novice, l'un dénonce la responsabilité de l'homme dans le réchauffement, l'autre rejette cette idée; de même, dans les textes de type expert, un texte souligne la responsabilité de l'homme, à l'inverse de l'autre texte; précisons que ce dernier texte, le texte 4 , manque d'objectivité de par le manque d'indépendance de l'auteur. Durant toute la séance de travail, les textes sont restés disponibles à l'ensemble des participants, de façon à approfondir l'exploitation des textes et à éviter le biais de la rétention d'information (c'est la compréhension en elle-même qui est évaluée dans cette étude, et non la mémorisation des informations).

Dans le groupe contrôle (sans guidage), les étudiants ont directement lu les textes tout en répondant au QCM, alors que, dans le groupe expérimental, ils ont utilisé le guidage 
pendant 10 minutes avant de répondre au questionnaire (sans surplus de temps). Les étudiants des deux groupes ont rendu le QCM 55 minutes après le début de la séance.

Pour analyser les données du QCM, trois catégories de questions ont été créées selon les processus cognitifs qu'elles induisent: les questions intertextuelles qui demandent de mettre en relation des informations venant de plusieurs textes, les questions source contenu qui demandent dans quel texte se trouve telle information, et les questions à inférence locale qui portent sur la compréhension de détails. Il était attendu que les lecteurs guidés répondent mieux que les autres au QCM (scores généraux) ainsi que dans ces trois catégories de questions, grâce au guidage qui repose sur une approche globale multidocumentaire.

Un tableau de codage attribuant un code spécifique à chaque catégorie de questions a permis de les distinguer (cf. Annexe 4), puis d'exploiter les données relatives à chaque catégorie. Certaines questions appartenaient à deux catégories à la fois (6 questions à inférence locale et source - contenu, 4 questions à inférence locale et intertextuelles).

Le pourcentage de bonnes réponses a été calculé pour chaque QCM rempli, il s'agit plus précisément du taux de bonnes réponses sur le nombre total de questions traitées (quelques questions n'ont pas été traitées chez une minorité de lecteurs), puis les moyennes et les écarts-types relatifs à chaque groupe expérimental ont été calculés ${ }^{3}$. Les performances au QCM ont été calculées en pourcentage pour les 19 questions à inférence locale et les 14 questions intertextuelles.

Pour les questions source - contenu, nous avons préféré observer le nombre de réponses correctes (nombre par participant, puis nombre moyen par groupe): comme ces questions sont moins nombreuses dans le QCM (10 au total), la quantité de bonnes réponses en nombre donne ici une idée plus précise et plus représentative des résultats (comparée au pourcentage); de plus, l'intégralité des questions source - contenu a été traitée par les participants, ce qui renforce l'intérêt de se baser sur des données en nombre pour cette catégorie de question. Enfin, un test $\mathrm{Chi}^{2}$, qui compare les proportions de bonnes réponses des deux groupes expérimentaux, a permis de savoir si l'un des groupes de lecteurs a significativement mieux répondu aux questions source - contenu.

Les synthèses rédigées par les participants ont, quant à elles, été analysées de la manière suivante : le nombre de références aux sources (termes faisant explicitement allusion à la source d'un texte: "dans le texte 1, " ou bien "Anna pense que...") a été compté dans chaque copie. Par ailleurs, les expressions qui regroupent plusieurs sources (exemple: " les textes 1 et 4 partagent la même opinion sur...") ont été repérées et comptées dans chaque synthèse car elles reflètent une compréhension transversale de la problématique.

\section{Résultats}

41 Il en ressort que les lecteurs guidés ont eu de meilleures performances au questionnaire (64\% de bonnes réponses, contre $59 \%$ dans le groupe contrôle) ainsi que dans les questions intertextuelles, les questions à inférence locale, et les questions source contenu (cf. Tableau 1). Pour cette dernière catégorie de question, un test $\mathrm{Chi}^{2}$ significatif (indice de probabilité supérieur à $95 \%$ ) confirme l'écart de performance entre le groupe guidé et le groupe contrôle.

42 Avec le guidage, les lecteurs n'ont globalement pas mieux évalué la crédibilité et l'objectivité des sources. L'évaluation de la crédibilité des documents était néanmoins 
bonne dans les feuilles de guidage, puisque le niveau d'expertise des documents et l'indépendance des auteurs y étaient pris en compte. Mais cette bonne évaluation des sources n'est pas ressortie par la suite dans le QCM : en y observant les réponses fournies sur les sources, on constate qu'il y a eu 38 bonnes réponses dans le groupe guidé (sur 64), et 42 dans le groupe contrôle (sur 64). Dans les deux groupes, le niveau d'évaluation des sources est correct ( $62 \%$ de bonnes réponses) mais reste assez moyen et n'a finalement pas été optimisé par le guidage.

43 Par ailleurs, les lecteurs guidés ont cité plus souvent les sources dans leur synthèse (15 références chez eux en moyenne, contre 11 dans le groupe contrôle), en faisant moins d'erreurs: il n'y en a aucune chez eux, tandis que dans le groupe sans guidage, il est arrivé deux fois qu'une information soit attribuée à la mauvaise source. De plus, les lecteurs guidés ont utilisé significativement plus d'expressions qui mentionnent plusieurs textes à la fois : ces expressions apparaissent en moyenne 3.37 fois dans les copies des guidés, contre 1.5 fois dans l'autre groupe. Ce résultat, qui ressort significativement dans un test statistique (test $\mathrm{T}$ ), suggère une meilleure compréhension des relations intertextuelles chez les lecteurs guidés.

Tableau 1 - Questionnaire à Choix Multiple : réponses correctes par type de question en pourcentage et en nombre moyen sur 10 .

\begin{tabular}{|l|c|c|}
\hline Type de question & Groupe guidé & Groupe contrôle : \\
\hline Questions à inférence locale & $64 \%$ & $56 \%$ \\
\hline Questions intertextuelles & $68 \%$ & $64 \%$ \\
\hline Questions source - contenu & $6,13 / 10$ & $4,38 / 10$ \\
\hline
\end{tabular}

\section{Conclusion}

Le guidage a eu tendance à favoriser la compréhension en général, mais aussi la compréhension détaillée, puisque les étudiants guidés ont obtenu plus de bonnes réponses aux questions qui impliquent une inférence locale. La meilleure compréhension des détails en condition guidée semble confirmer l'utilité de l'approche globale (Moirand, 1979 ; Bondrea, 2011).

Le guidage a aussi favorisé la compréhension intertextuelle. Cela s'observe à plusieurs niveaux : dans le QCM, les questions qui demandent au lecteur de relier les sources à leur contenu ont mené à de meilleurs résultats chez les lecteurs guidés. Cette meilleure intégration du "qui dit quoi ?" a sans doute permis, par la suite, une meilleure compréhension intertextuelle dans les synthèses : en effet, les lecteurs qui ont bénéficié de notre outil méthodologique citent plus souvent les sources dans leurs copies et ont une vision plus transversale et plus synthétique de la problématique (références aux sources groupées).

L'intégration de la représentation mentale multi-documentaire, étape nécessaire selon Rouet (2006), a donc pu être facilitée par notre outil pédagogique. De plus, par une première approche globale des textes, l'outil a probablement permis une meilleure 
régulation de la charge cognitive pendant la lecture. Les lecteurs auraient alors eu plus de ressources cognitives pour comprendre les textes de manière à la fois transversale et détaillée.

Cette étude renforce l'idée du rôle clé de la compréhension des sources dans la lecture multidocumentaire : une compréhension plus complète (variété des points de vue) et plus transversale de la problématique en découlerait (Rouet, 2006; Strømsø, Bråten \& Britt, 2009 ; Gebril \& Plakans 2009).

L'analyse des sources n'a pas vraiment été optimisée par notre outil pédagogique (d'après les résultats aux questions sur les sources dans le QCM) : il semblerait qu'un travail de fond à plus long terme soit nécessaire pour que le lecteur adopte un regard plus critique sur la crédibilité et l'objectivité des documents. Une analyse explicitée des sources, au moment de la correction, pourrait alors compléter le guidage (cela pourrait être testé dans une future étude de long terme).

49 Cependant, le travail sur les sources, exigé dès le début de la lecture grâce au guidage, a sans doute contribué à ce que les lecteurs guidés distinguent mieux les textes entre eux et soient plus attentifs à l'origine des informations lues puisqu'ils citent plus souvent les sources dans leur synthèse, en se trompant moins. On peut aussi supposer que le guidage a sensibilisé les lecteurs à l'utilité de préciser l'origine de l'information dans leurs copies (ce qui a déjà son utilité sur le plan pédagogique).

50 Notre étude rappelle aussi que la lecture en langue seconde nécessite une intervention pédagogique, même chez les étudiants de niveaux avancés, pour une compréhension des textes plus en détail et en nuance (Gaonac'h, 2005 ; Smuk, 2011). Cette intervention serait particulièrement pertinente lorsqu'elle fait appel, chez le lecteur, à des stratégies globales en première phase de lecture (Moirand, 1979; Chen \& Graves, 1995 ; Plakans, 2009 ; Huang, Chern, \& Lin, 2009 ; Kai, 2011 ; Bondrea, 2011).

51 En ce qui concerne la méthode de recherche, nous retenons l'intérêt d'élaborer des matériels tels que notre QCM qui permet d'évaluer différents types de compréhension (compréhension détaillée, compréhension intertextuelle, compréhension des liens entre sources et contenus). Face à la difficulté d'évaluer la compréhension des textes en langue seconde, il semble également pertinent d'avoir plusieurs cordes à son arc, c'est-à-dire de mesurer la compréhension à travers l'évaluation de différentes tâches de compréhension: en s'appuyant de façon complémentaire sur divers indicateurs, nous pouvons les recouper pour savoir s'ils convergent ou non vers les mêmes tendances.

Dans notre étude, différents types d'indicateurs de compréhension ont ainsi été recherchés. En effet, les résultats du QCM (performances générales, performances par type de question), combinés aux références aux sources dans les synthèses (quantité totale, quantité sous forme groupée) nous ont aidés à mieux évaluer l'impact de notre guidage sur la compréhension de documents multiples en langue seconde.

Enfin, notre approche a été pluridisciplinaire ${ }^{4}$ : les références théoriques qui ont servi de socle à la conception du guidage relèvent de la didactique des langues, de la psycholinguistique et de la psychologie cognitive. Par ailleurs, notre démarche expérimentale a reposé sur une approche de psychologie cognitive tout en s'inscrivant dans une situation pédagogique réelle. À travers cette étude, nous espérons donc mettre en lumière l'intérêt d'une approche pluridisciplinaire pour mieux cerner le fonctionnement de l'apprentissage des langues et pour proposer des solutions pédagogiques adaptées aux besoins des apprenants. 


\section{BIBLIOGRAPHIE}

Acuňa, T. (2005). "Le passage des processus de bas niveau aux processus de haut niveau dans l'élaboration d'une représentation du texte". Acquisition et interaction en langue étrangère. Disponible en ligne. http://aile.revues.org/356

Alptekin, C., \& Erçetin, G. (2009). "Assessing the relationship of working memory to L2 reading: Does the nature of comprehension process and reading span task make a difference?" System, vol. $37, \mathrm{n}^{\circ} 4$, pp. 627-639.

Bernhardt, E. (1987). "Cognitive processes in L2: an examination of reading behaviors". In Lantolf, J. \& Labarca, A. (dir.). Research on Second Language Acquisition in classroom settings. Norwood, NJ: Ablex, pp. 35-51.

Block, E. (1986). "The comprehension strategies of second language readers". TESOL Quarterly, vol. 20, n³, pp. 463-494.

Bondrea, E. (2011). « Lire en français des articles de presse à l'usage d'un public spécifique : réflexions didactiques et linguistiques ». In R. Redouane (dir.), Regards croisés sur l'enseignement des langues étrangères. L'Harmattan, pp. 213-229.

Borau, V. (2012). "Enseigner le français sur objectifs universitaires". Le Français Dans le Monde, n - 383 , pp. 36-37.

Charles-Dominique, G. (2012). Lire et utiliser des textes en L2 et langue seconde : l'impact du guidage de la lecture sur la compréhension. Thèse de doctorat en psychologie, Université de Poitiers.

Chen, H.-C. \& Graves, M.F. (1995). "Effects of previewing and providing background knowledge on Tawainese college students' comprehension of American short stories". TESOL Quarterly, vol. 29, pp. 663-686.

Coirier, P., Gaonac'h, D. \& Passerault, J.-M. (1996). Psycholinguistique textuelle. Approche cognitive de la compréhension et de la production des textes. Paris : Armand Colin.

Gaonac'h, D. (2005). "La lecture en langue étrangère : un tour d'horizon d'une problématique de psychologie cognitive". Acquisition et interaction en langue étrangère. Disponible en ligne. http:// aile.revues.org/970 REF INCOMPLETE

Garcia-Debanc, C. (1989). "Le tri de textes : modes d'emploi". Pratiques, vol. 62, pp. 3-51.

Gebril, A. \& Plakans, L. (2009). "Investigating Source Use, Discourse Features, and Process in Integrated Writing Tests". Spaan Fellow Working Papers in Second or Foreign Language Assessment, vol. 7, pp. 47-84. Disponible en ligne.

http://www.cambridgemichigan.org/sites/default/files/resources/SpaanPapers/ Spaan_V7_GebrilPlakans.pdf

Huang, H.C., Chern, C.L. \& Lin, C.C. (2009). "EFL learners' use of online reading strategies and comprehension of texts: An exploratory study". Computers \& Education, vol. 52, n 1, pp. 13-26.

Kai, A. (2011). "Effects of Generative Tasks on Japanese EFL Readers' Metacomprehension Accuracy". Poster présenté au colloque Society for Text and Discourse, Poitiers (France), 11-13.07.2011. 
Limón, M. \& Vidal, S. (2011). "Use of Sources and Learning from Multiple Documents: Does Individuals' Epistemic Competence Influence them?". Poster présenté au colloque Society for Text and Discourse, Poitiers (France), 11-13.07.2011.

Meissner, F. J., Meissner, C., Klein, H. G., \& Stegmann, T. D. (2004). EuroComRom. Lire les langues romanes dès le départ. Avec une introduction à la didactique de l'eurocompréhension. Aachen : Shaker.

Moirand, S. (1979). Situations d'écrit. Paris : Clé international.

Olive, T. \& Piolat, A. (2011). "Students' comprehension and cognitive effort when taking notes and composing from a source text: Effects of formatting". Colloque Society for Text and Discourse, Poitiers (France), 11-13.07.2011.

Payne, T. W., Kalibatseva, Z., \& Jungers, M. K. (2009). “Does domain experience compensate for working memory capacity in second language reading comprehension?" Learning and Individual Differences, vol. 19, pp. 119-123.

Plakans, L. (2009). "The role of reading strategies in integrated L2 writing tasks". Journal of English for Academic Purposes, pp. 1-15. Disponible en ligne.

http://www.esoluk.co.uk/digibooks/pdfs/L2_reading_writing.pdf

Rouet, J.-F. (2006). The Skills of Document Use: From Text Comprehension to Web-Based Learning. Mahwah, NJ: Lawrence Erlbaum Associates.

Rouet, J.-F., \& Britt, M.A. (2011). "Relevance processes in multiple document comprehension". In McCrudden, M.T., Magliano, J. P. \& Schraw, G. (dir.). Text Relevance and Learning from Text. Greenwich, CT: Information Age Publishing, pp. 19-52.

Rouet, J.-F., Vidal-Abarca, E., Bert-Erboul, A. \& Millogo, V. (2001). "Effects of information search tasks on the comprehension of instructional text". Discourse Processes, vol. 31, n 2, pp. 163-186.

Smuk, M. (2011). "Les stratégies métacognitives et le développement des compétences liées à l'expression écrite en fonction du niveau des apprenants". Etude présentée au colloque Les compétences en progression : défi pour la didactique des langues, Poitiers (France), 23-24.09.2011.

Strømsø, H.I., Bråten, I., \& Britt, M.A. (2009). "Reading multiple texts about climate change: The relationship between memory for sources and text comprehension". Learning and Instruction, vol. 20, pp. 192-204.

Wahl, Y. (2001). "L'évaluation de la compréhension en lecture : une mission impossible ?". In Didactique des langues romanes, le développement de compétences chez l'apprenant. Actes du colloque de Louvain-la-Neuve, 27-29 janvier 2000. Bruxelles : De Boeck, pp. 583-590.

\section{ANNEXES}

\section{Annexe 1}

Outil pédagogique testé : Guidage pour la lecture multidocumentaire en langue seconde 


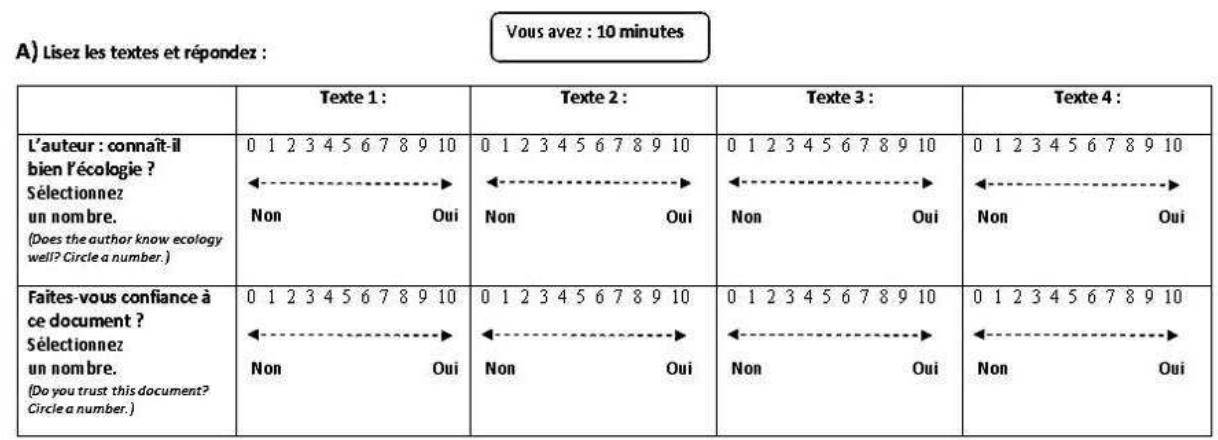

B) Reliez chaque idée avec le texte qui correspond (sélectionnez 1 seule réponsel. Attention : il y a 2 idées qui ne sont pas dans les textes.

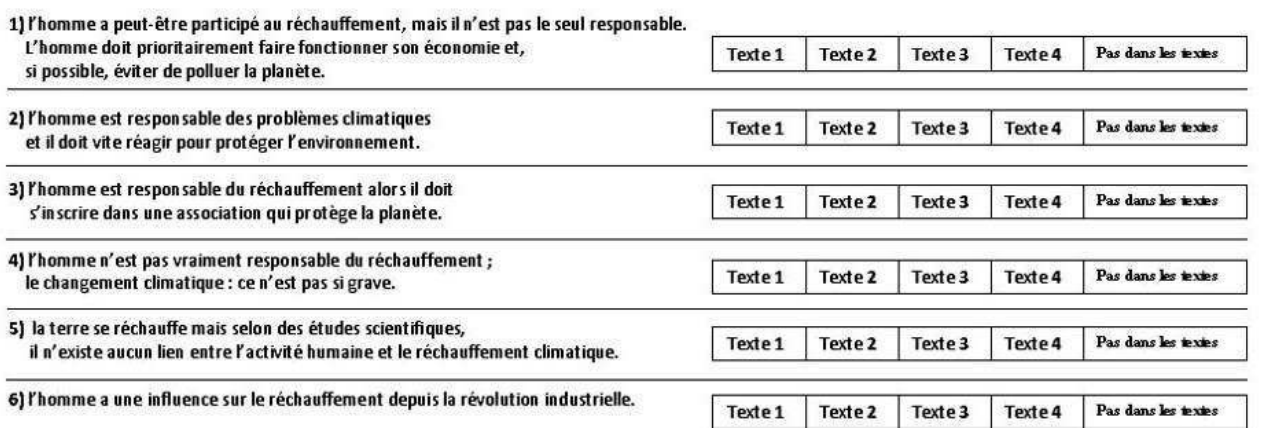

Annexe 2 Textes à lire

Sur internet, ces quatre textes abordent le thème du réchauffement climatique.

Texte 1:

Site : Réactu : le blogue de notre actu.

Anna :

Bonjour,

Hier, on m'a parlé d'une histoire de mails de chercheurs interceptés : en gros, le problème du réchauffement serait carrément exagéré. Alors j'en profite pour vous dire que moi aussi j'ai l'impression qu'on en fait trop, et puis j'en ai un peu assez qu'on nous culpabilise sur ce thème. D'abord, j'ai du mal à croire que l'homme ait pu à lui tout seul dérégler le climat (dans quel sens d'ailleurs? Avec cet hiver glacial, je me le demande...). Un jour, j'ai même vu à la télé qu'il y a eu dans le passé une période où il faisait plus chaud qu'aujourd'hui : pas de quoi paniquer jusque-là.

Je dois dire que le climat, c'est pas ma spécialité mais logiquement, ce n'est pas quelques degrés de plus qui vont détruire la planète. Et même si l'homme était le grand responsable : doit-il s'excuser de vivre sur sa planète et ralentir son économie (qui n'est déjà pas au beau fixe) ? En plus, c'est trop facile de dire « stop on arrête tout » aux autres pays, ils ont eux aussi le droit de se moderniser... Une chose est sûre : je ne veux plus voir de reportages télé sur le climat car c'est déprimant ! C'est vrai qu'il y a des endroits de la planète qui connaissent des problèmes (la banquise...) mais ailleurs, si on regarde autour de nous, la nature est encore là, toujours aussi belle, et elle fourmille d'animaux (OK, on a moins d'abeilles qu'avant mais on peut bien vivre sans !).

J'ai le sentiment d'être la seule à dire non à ce catastrophisme ambiant. Y en a-t-il d'autres qui pensent comme moi ? Sinon, moi je reste ouverte au débat mais j'attends d'être convaincue... 
Texte 2:

Même site, réponse à Anna.

Ben :

Ce n'est pas du catastrophisme, c'est plutôt en réagissant comme ça qu'on court à la catastrophe!

Avec le mari de ma sœur qui est météorologue et mon association « terre en danger », je connais bien ce problème de réchauffement. Par exemple, je suis en contact avec des gens du monde entier qui nous décrivent ce à quoi ils assistent : des feux de forêt toujours plus meurtriers, des glaciers qui fondent, des rivières qui débordent...

Oui, l’homme récolte ce qu'il a semé ! Et « Oui l'homme à lui tout seul a pu faire ça » car la terre repose sur un équilibre fragile (je note au passage que l'abeille y a sa place). Il faut réagir dès maintenant pour essayer de le préserver. S'il y a une chose qui m'agace, c'est bien l'argument à deux sous « on a un hiver froid ». J'ai deux réponses à cette absurdité :

1- Ça n'empêche pas d'avoir des étés caniculaires.

2- Là, on est sur une petite échelle, ce qu'il faut voir, c'est que la planète se réchauffe à une grande échelle.

Ce discours va typiquement dans le sens de ceux qui ne veulent pas changer leurs habitudes (l'histoire des mails piratés tombe à pique...). De toute façon, il est évident que derrière ces rumeurs de réchauffement imaginaire, il y a les lobbies industriels.

Quand j'ai lu le message d'Anna, je n'ai pas pu m'empêcher de réagir, mais franchement, il n'y a pas matière à débat, les faits sont là : alors, ouvre les yeux, Anna, même si c'est un peu dur de voir la réalité en face !

Texte 3 :

Site : Ecologia, un site de vulgarisation scientifique sur l'environnement proposé par le Centre National de Recherche Scientifique Français (CNRSF).

Foire aux Questions (FAQ) : nos chercheurs répondent aux internautes.

En quoi consiste ce que l'on appelle le réchauffement climatique?

Ce phénomène global a été constaté à travers l'augmentation de la température de l'air et de l'eau. Les données ne sont pas forcément spectaculaires pour des néophytes puisqu'il ne s'agit que d'une légère hausse dans la moyenne des températures, mais cela peut néanmoins suffire à dérégler certains équilibres écologiques.

Quelles sont les conséquences du réchauffement?

D'une part, les catastrophes naturelles (incendies, inondations, tempêtes) ont tendance à être plus fréquentes et touchent même des zones géographiques habituellement épargnées. D'autre part, on constate simultanément un assèchement des lacs ou des rivières et une montée des eaux (due à la fonte des glaces) qui peut, quant à elle, élargir des rivières, créer de nouveaux lacs ou bien faire disparaître des îlots. Près du cercle polaire, par exemple, les routes migratoires des caribous ont été coupées par les eaux. Cela a aussi eu des répercussions sur les populations qui chassaient d'habitude cet animal pour se nourrir.

Quel est le rôle de l'homme dans le réchauffement? 
Depuis le début de l'industrialisation, l'intensification des activités humaines est accompagnée d'un réchauffement global. Le rapport du Giec de 2001 établit ainsi ce lien homme - réchauffement avec une probabilité d'au moins $66 \%$. La vie moderne de l'homme implique généralement le rejet de gaz à effet de serre (dont le CO2) dans l'atmosphère. Or nous savons que ces gaz renforcent le réchauffement. Il est alors crucial de prendre en compte le rôle de l'homme dans les changements climatiques.

Nous poursuivons par ailleurs des recherches afin d'obtenir des conclusions plus précises concernant l'impact des activités humaines sur l'environnement.

Que signifient les hivers froids que la France a connus ces deux dernières années ? Ils sont certes paradoxaux à l'heure du réchauffement mais ne contredisent pas nos observations : ainsi, le mois de janvier 2010, qui fut très froid en France, a cependant été le plus chaud depuis plus de 40 ans si l'on se fie aux moyennes des températures à un niveau mondial. Les hivers prochains nous donneront plus de recul afin de savoir si ces « coups de froid» localisés représentent une nouvelle donne ou non.

\section{Texte 4:}

Site du journal : Actualités \& Economie

L'homme a-t-il semé le réchauffement climatique?

Robert Lontier, directeur de l'Institut des Etudes sur la Géochimie et l'Industrie (IEGI), nous a accordé une interview pour répondre à cette question.

A \& E* : Pensez-vous que l'homme soit responsable du réchauffement climatique?

R. L. ** : Je pense que les activités humaines ont évidemment un certain impact sur l'environnement mais qui reste toutefois assez négligeable comparé à d'autres phénomènes bien plus importants. Je fais ici allusion à l'influence du soleil sur la planète : nous savons que ses rayons la réchauffent de façon croissante. Il est hélas très difficile de mesurer ces données à une grande échelle mais il semble que le rôle du soleil ne soit pas à négliger.

A \& E : D'après vous, c'est le soleil, pourtant si loin et si vieux, qui est la cause d'un tel changement climatique?

R. L. : Oui. Cette étoile est éloignée de la terre mais la chaleur qu'elle dégage, de part la puissance de son activité, n'en est pas moins forte. Par ailleurs, je tiens à relativiser ce que vous appelez « un tel changement climatique » : les études en climatologie évoquent des épisodes chauds dans le passé, notamment dans une période moyenâgeuse qui a été plus chaude encore qu'aujourd'hui.

A \& E : Votre point de vue semble plutôt marginal, qu'avez-vous à dire là-dessus ?

R. L. : Ma position est aujourd'hui assez rare mais de moins en moins d'après mes observations. De plus, ce « consensus scientifique » n'est pas un argument : il est ainsi arrivé dans le passé que certains scientifiques découvrent la vérité contre l'avis général de leur époque (ce fut le cas pour la rotation de la terre, la dérive des continents...). Le débat doit exister et mes propos -publiés, je précise- y ont toute leur place.

A \& E : En attendant, que peut faire l'homme face au problème climatique?

R. L. : Il doit évidemment économiser ses énergies ou ressources naturelles et éviter de polluer la planète afin de préserver son avenir. Mais il ne faut pas rentrer dans une 
logique qui impose de nouvelles normes trop strictes ou qui bannisse nos énergies habituelles car cela pourrait nuire à nos industries, sources d'emplois. Je pense que face au réchauffement, l'homme peut trouver des solutions sans pour autant causer sa ruine économique.

* A \& E = le journaliste d'Actualités et Economie

** R. L. = Robert Lontier

Annexe 3 QCM

Pour Anna : (1 réponse)

$\square$ s'il fait un peu plus chaud, c'est bien parce qu'elle a eu trop froid cet hiver.

$\square$ s'il fait un peu plus chaud, ce n'est pas grave. Par exemple, il a fait encore plus chaud il y a longtemps dans le passé.

$\square$ quelques degrés de plus peuvent détruire la planète mais ce n'est pas l'homme qui en est responsable.

$\square$ je ne sais pas.

Pour Anna, l'homme... (1 réponse)

$\square$ devrait s'excuser parce qu'il est peut-être le grand responsable.

$\square$ est peut-être responsable du réchauffement, mais il ne doit pas limiter son activité économique et il peut continuer de vivre comme il le fait.

$\square$ est peut-être responsable du réchauffement, alors il devrait ralentir son économie.

$\square$ doit faire accélérer son économie : il faut produire plus car on est dans une crise économique.

$\square$ je ne sais pas.

Pour Anna, les autres pays... (1 réponse)

$\square$ doivent arrêter leur développement économique, même si c'est difficile.

$\square$ aimeraient se moderniser, mais le réchauffement peut ralentir l'économie.

$\square$ devraient développer leur économie, même si ce n'est pas très bon pour l'écologie.

$\square$ devraient ralentir leur économie parce que c'est mieux pour l'écologie.

$\square$ je ne sais pas.

Que pense Anna du réchauffement ? (1 réponse)

$\square$ il provoque quelques problèmes mais dans l'ensemble, la nature va bien.

$\square$ si on regarde la nature : il y a plein d'animaux, il y a beaucoup d'abeilles et c'est toujours aussi beau.

$\square$ il y a des problèmes graves qui concernent par exemple la banquise et les abeilles, et c'est déprimant de voir toute la nature dans cette situation.

$\square$ je ne sais pas.

Ben : quel est son comportement face au message d'Anna ? Il est...(1 réponse)

$\square$ amusé et intéressé. 
critique mais tolérant et ouvert au débat.

agacé et critique.

$\square$ critique en général, mais d'accord avec Anna sur certains détails (les abeilles...).

il n'a pas vraiment voulu réagir car il n'y a pas matière à débat.

$\square$ je ne sais pas.

Ben « connaît bien ce problème de réchauffement » climatique parce que... (1 réponse)

$\square$ son beau-frère, qui est météorologue, dirige l'association « terre en danger ».

$\square$ il est en relation avec des gens qui s'intéressent aux phénomènes climatiques et il est engagé dans une association écologiste.

$\square$ il connaît des gens qui ont vu des catastrophes naturelles dans toute la France.

$\square$ il est dans une association de météorologues qui échangent leurs connaissances sur le réchauffement.

il a lui-même vu les conséquences du réchauffement : des feux de forêt, des glaciers qui fondent, des rivières qui débordent..

$\square$ je ne sais pas

Selon Ben, il est possible que l'homme soit responsable du réchauffement parce que : (1 réponse)

$\square$ une petite abeille peut détruire la forêt.

l'homme pollue l'eau, l'air et la terre qui sont alors devenus plus fragiles.

$\square$ l'équilibre de l'homme est fragile.

$\square$ une petite action sur l'environnement peut fragiliser le fonctionnement de la planète.

$\square$ la pollution industrielle est sûrement plus importante que ce que l'on croit.

$\square$ je ne sais pas

Pour Ben, le discours selon lequel le réchauffement climatique n'est pas un vrai problème, c'est le discours... (1 réponse)

$\square$ des lobbies écologistes.

$\square$ des gens qui ne sont pas assez informés.

$\square$ des gens qui refusent de faire des efforts ou qui protègent les intérêts industriels.

$\square$ des gens qui veulent changer leurs habitudes mais qui n'y arrivent pas (c'est trop difficile).

$\square$ des gens qui ne pensent qu'à eux-mêmes et de quelques hommes politiques.

$\square$ je ne sais pas

Texte 3 : dans cette liste, 2 phénomènes ne sont pas des conséquences du réchauffement. Quelles sont ces 2 erreurs ? (donner 2 réponses).

$\square$ les incendies. 
$\square$ l'eau qui monte.

$\square$ des lacs et des îlots qui peuvent se créer ou disparaître.

$\square$ les caribous qui ne peuvent plus boire dans la rivière.

les tempêtes.

$\square$ les migrations d'animaux qui sont modifiées.

$\square$ les iceberg qui fondent au soleil.

$\square$ les routes migratoires qui sont coupées par les eaux dans les pays chauds.

$\square$ des rivières qui deviennent plus larges.

$\square$ je ne sais pas.

D'après vous, qui dit qu'il existe un lien entre l'homme et le réchauffement ? (1 réponse)

$\square$ un collègue chercheur (Monsieur Giec)

$\square$ un rapport scientifique

$\square$ le site web Ecologia

$\square$ un rapport du Groupe de l'Industrie Et du Commerce

$\square$ je ne sais pas.

Selon le texte 3, est-ce que l'homme est responsable du réchauffement ? (1 réponse)

$\square$ non, mais il faut continuer les recherches pour en savoir plus.

$\square$ oui, un peu. Une partie du problème est liée à l'homme, l'autre partie aux animaux.

$\square$ oui, l'homme en serait majoritairement la cause.

$\square$ oui, mais seulement à $66 \% .34 \%$ est causé par l'industrie.

$\square$ oui, mais le rôle de l'homme serait minoritaire.

$\square$ oui, parce que des liquides (eau polluée...) sont rejetés dans l'atmosphère.

$\square$ je ne sais pas.

Selon le texte 3 , les hivers froids... (1 réponse)

$\square$ montrent que la terre ne se réchauffe pas vraiment. C'est aussi ce que disait Anna (texte 1)

c'est vrai qu'ils peuvent être froids. Cependant, sur une longue période et sur toute la terre, on observe un réchauffement de la planète. C'est une question d'échelle, comme le disait Ben (texte 2).

$\square$ ils peuvent être froids en 2010 mais ils étaient très chauds dans le passé. Le texte 4 exprime la même idée et explique cela par l'activité du soleil.

ils sont paradoxaux à l'heure du réchauffement. Les hivers prochains permettront alors de savoir si oui ou non la terre se réchauffe. Et dans le texte 1, Anna se demande même si il est possible que la terre se refroidisse.

$\square$ je ne sais pas. 


\section{Texte 4 : selon Robert Lontier, l'homme est-il responsable du réchauffement climatique? (1 réponse)}

$\square$ oui, c'est le seul responsable.

$\square$ oui, son impact sur le climat est aussi fort que le soleil.

$\square$ oui, un peu.

$\square$ non, parce que le soleil est le seul responsable.

$\square$ oui, mais c'est négligeable à côté des phénomènes aquatiques.

$\square$ oui, beaucoup plus que le soleil.

$\square$ je ne sais pas.

D'après Robert Lontier, dans le passé... (1 réponse)

$\square$ il n'y a pas eu de changement climatique.

$\square$ il y a eu une période très froide (au moyen âge), puis le climat est devenu de plus en plus chaud.

$\square$ il a fait chaud, de plus en plus chaud, et il fait encore plus chaud aujourd'hui : c'est un vrai changement climatique.

$\square$ il a fait chaud mais pas autant qu'aujourd'hui (le changement climatique actuel est donc inquiétant).

$\square$ il a fait chaud, puis ça s'est refroidi, et aujourd'hui cela se réchauffe encore : ces changements sont normaux.

$\square$ je ne sais pas.

Pourquoi Robert Lontier parle-t-il de la « rotation de la terre » ? (1 réponse)

$\square$ parce que la terre tourne et cela fait augmenter sa température.

$\square$ parce qu'il a spécifiquement étudié le problème de la rotation de la terre.

$\square$ parce que c'est une grande découverte qu'il a faite dans le passé.

$\square$ parce que, à l'époque, personne ne savait que la terre chauffe au point de rôtir.

$\square$ parce que le scientifique qui l'a découverte était seul à penser que la terre tourne.

$\square$ parce que dans le passé, la majorité des scientifiques a découvert que la terre tournait.

$\square$ parce que la terre est dans la même situation que le soleil : elle subit une rotation.

$\square$ je ne sais pas.

De quoi Robert Lontier a-t-il peur ? (1 réponse)

$\square$ que l'homme pollue la planète en utilisant ses énergies habituelles.

$\square$ que l'homme dépense trop d'argent si il y a des normes écologiques trop strictes.

$\square$ que l'homme crée des règles plus écologiques qui aient un impact négatif sur ses industries.

$\square$ que le réchauffement ralentisse l'économie et fasse baisser le nombre d'emplois. 
$\square$ que l'homme rentre dans une logique écologiste : il peut utiliser ses énergies habituelles sans se limiter.

$\square$ je ne sais pas.

Dans quel texte est-on le plus inquiété par le réchauffement ? Trouvez le bon ordre $(+\rightarrow-)$. (Which text expresses the most worry about global warming? Find the right order.)

$\square$ 1- Texte 4 / 2- Texte 2 / 3- Texte 3 / 4- Texte 1.

1- Texte 1 / 2-Texte 3 / 3- Texte $2 / 4$ - Texte 4.

1- Texte 2 / 2- Texte 3 / 3- Texte 4 / 4- Texte 1 . (1 seule réponse)

1- Texte 2 / 2-Texte 4 / 3- Texte 1 / 4- Texte 3.

1- Texte 2 / 2- Texte 4 / 3- Texte 3 / 4- Texte 1.

$\square$ je ne sais pas.

De manière directe ou indirecte, dans quel texte est-ce qu'on critique...

\begin{tabular}{|l|c|l|l|l|l|}
\hline & Texte 1 & $/ 2 /$ & $/ 3 /$ & $/ 4 /$ & pas dans les textes \\
\hline - la supériorité de l'opinion générale & $\square$ & $\square$ & $\square$ & $\square$ & $\square$ (1 réponse) \\
\hline - le pessimisme à propos du réchauffement? & $\square$ & $\square$ & $\square$ & $\square$ & $\square$ (1 réponse) \\
\hline - les lois très écologiques? & $\square$ & $\square$ & $\square$ & $\square$ & $\square$ (1 réponse) \\
\hline - les lobbies industriels? & $\square$ & $\square$ & $\square$ & $\square$ & $\square$ (1 réponse) \\
\hline - les scientifiques du passé ? & $\square$ & $\square$ & $\square$ & $\square$ & $\square$ (1 réponse) \\
\hline - les autres pays? & $\square$ & $\square$ & $\square$ & $\square$ & $\square$ (1 réponse) \\
\hline - les gens qui ont peur de la réalité ? & $\square$ & $\square$ & $\square$ & $\square$ & $\square$ (1 réponse) \\
\hline - les reportages sur le climat? & $\square$ & $\square$ & $\square$ & $\square$ & $\square$ (1 réponse) \\
\hline - les gens qui mangent du caribou? & $\square$ & $\square$ & $\square$ & $\square$ & $\square$ (1 réponse) \\
\hline - l'existence d'un débat sur le réchauffement? & $\square$ & $\square$ & $\square$ & $\square$ & $\square$ (1 réponse) \\
\hline
\end{tabular}

Pour chaque sujet, dites si les textes ont une opinion identique ou différente :

\begin{tabular}{|l|c|c|c|}
\hline Sujet & Textes & $\begin{array}{c}\text { Opinions } \\
\text { identiques }\end{array}$ & / différentes \\
\hline L'abeille & 1 et 2 & $\square$ & $\square$ \\
\hline Les hivers froids & 1 et 2 & $\square$ & $\square$ \\
\hline
\end{tabular}




\begin{tabular}{|l|c|c|c|}
\hline Les hivers froids & 2 et 3 & $\square$ & $\square$ \\
\hline L'économie = la priorité ? & 1 et 4 & $\square$ & $\square$ \\
\hline $\begin{array}{l}\text { L'équilibre de la planète } \\
\text { = fragile, facilement affecté ? }\end{array}$ & 1 et 2 & $\square$ & $\square$ \\
\hline $\begin{array}{l}\text { L'équilibre de la planète } \\
\text { = fragile, facilement affecté ? }\end{array}$ & 2 et 3 & $\square$ & $\square$ \\
\hline Il faut préserver et respecter la planète & 2 et 4 & $\square$ & $\square$ \\
\hline
\end{tabular}

\begin{tabular}{|l|c|c|c|}
\hline Sujet & Textes & $\begin{array}{c}\text { Opinions } \\
\text { identiques }\end{array}$ & / différentes \\
\hline $\begin{array}{l}\text { L'homme = principale cause } \\
\text { du réchauffement climatique? } \\
\text { (human= the main reason for global warming?) }\end{array}$ & 1 et 2 & $\square$ & $\square$ \\
\hline $\begin{array}{l}\text { L'homme = principale cause } \\
\text { du réchauffement climatique? }\end{array}$ & 3 et 4 & $\square$ & $\square$ \\
\hline $\begin{array}{l}\text { L'homme = principale cause } \\
\text { du réchauffement climatique? }\end{array}$ & 1 et 4 & $\square$ & $\square$ \\
\hline $\begin{array}{l}\text { L'homme = principale cause } \\
\text { du réchauffement climatique? }\end{array}$ & 2 et 3 & $\square$ & $\square$ \\
\hline $\begin{array}{l}\text { Le soleil = la première cause } \\
\text { du réchauffement climatique? }\end{array}$ & 2 et 4 & $\square$ & $\square$ \\
\hline
\end{tabular}

D'après vous, les documents sont-ils sérieux ?

Sélectionnez un nombre pour chaque texte

(Circle a number for each text

\begin{tabular}{|l|lllll|}
\hline & Pas du tout sérieux & très sérieux \\
\hline Texte 1 & 1 & 2 & 3 & 4 & 5 \\
\hline Texte 2 & 1 & 2 & 3 & 4 & 5 \\
\hline
\end{tabular}

D'après vous, les documents

sont-ils objectifs (points de vue neutres)?

Sélectionnez un nombre pour chaque texte 
(Circle a number for each text).

\begin{tabular}{|l|llllll|}
\hline & Pas du tout objectif & très objectif \\
\hline Texte 1 & 1 & 2 & 3 & 4 & 5 \\
\hline Texte 2 & 1 & 2 & 3 & 4 & 5 \\
\hline
\end{tabular}

Annexe 4 Codage des questions selon leur catégorie

Questions à inférence locale (notées IL) :

$\mathrm{IL}=$ réponses $\mathrm{n}^{\circ} 2,3,5,6,7,12,14,15,16,20,21,23,24,25,27,30,32,35$, et 38.

Questions intertextuelles (notées B) :

$\mathrm{B}=$ réponses $\mathrm{n}^{\circ} 13,18,29,30,31,32,33,34,35,36,37,38,39$, et 40.

Questions "source-contenu" (notées SC) :

$\mathrm{SC}=$ réponses $\mathrm{n}^{\circ} 19,20,21,22,23,24,25,26,27$, et 28.

Annexe 5 Réponses attendues au QCM

Remarque : quand un numéro de question (ou un numéro de réponse) est précisé cidessous, il s'agit simplement de l'ordre de présentation de la question (ou de la réponse) dans le QCM.

Question 1- réponse 2 (noté aussi : Q1-R2), Q2-R2, Q3-R3, Q4-R1, Q5-R3, Q6-R2, Q7-R4, Q8$\mathrm{R} 3, \mathrm{Q} 9 / \mathrm{Q} 10$ (2 réponses attendues ici) - R4 et 8., Q11-R2, Q12-R3, Q13-R2, Q14-R3, Q15-R5, Q16-R5, Q17-R3, Q18-R3,

Q19- Texte 4 (ou bien T4), Q20-T1, Q21-T4, Q22-T2, Q23- Hors texte (ou bien HT), Q24-HT, Q25-T2, Q26-T1, Q27-HT, Q28- T2,

Q29-diff., Q30-diff., Q31-id., Q32-id., Q33-diff., Q34-id., Q35-id., Q36-diff., Q37-diff., Q38-id., Q39-id., Q40-diff.,

Q41-1 ou 2 accepté, Q42-1 ou 2 accepté, Q43- 4 ou 5 accepté, Q44- 4 ou 5 accepté, Q45- 1 ou 2 accepté, Q46- 1 ou 2 accepté, Q47-5, Q48- 1, 2 ou 3 accepté.

\section{NOTES}

1. Outil au sens de support technique (une feuille A4 ici) combiné à une certaine approche ecture.

2. Nous rattacherons à la notion de source tout ce qui peut référer à l'origine du document: l'auteur (reconnaissable par son nom, sa profession, sa nationalité ou d'autres indices qui permettent de le distinguer), le site web (ou le journal, la revue), la date de parution, ou encore le numéro du texte si un numéro lui est attribué.

3. Notons ici l'intérêt des tests statistiques, utilisés dans les études de psycholinguistique et de psychologie cognitive : ils permettent d'indiquer le caractère significatif (ou non significatif) d'un résultat. Pour comparer les performances de deux groupes expérimentaux, la moyenne de chaque groupe est une donnée utile mais qui ne reflète pas les variations au sein de chaque 
groupe. Alors, un approfondissement statistique (tel que le "test $\mathrm{T}$ ") prend en compte ces variations : en vérifiant l'homogénéité des données dans chaque groupe, il confirme la validité de l'écart entre les moyennes. Les tests statistiques fournissent par ailleurs un indice de probabilité : s'il est de $95 \%$ (noté aussi .05), cela signifie qu'en reproduisant l'étude, nous aurions $95 \%$ de chances d'obtenir un tel écart entre les deux groupes.

4. Cette étude s'est déroulée dans le cadre d'un doctorat pluridisciplinaire (direction au sein d'un laboratoire de psychologie, le CeRCA, et co-direction dans un laboratoire axé sur les sciences du langage, le FORELL, à Poitiers) ; parallèlement, la doctorante s'est nourrie de son expérience d'enseignante en français langue étrangère et seconde. Pour plus de détails sur l'étude, vous pouvez consulter sa thèse TITRE ? (Charles-Dominique, 2012).

\section{RÉSUMÉS}

Des connaissances issues de la didactique des langues, de la psycholinguistique et de la psychologie cognitive ont permis de concevoir un outil pédagogique qui guide la lecture de textes en français langue seconde. Cet outil méthodologique a été créé dans le but d'améliorer la compréhension des liens entre les textes (points communs ou divergences entre les quatre textes à lire), la compréhension des sources en lien avec leur contenu (qui dit quoi) et enfin, la compréhension des détails. Les résultats de l'étude tendent à renforcer certaines théories sur la lecture en langue seconde et débouchent sur des considérations pédagogiques.

In this study theories of language didactics, psycholinguistics and cognitive psychology supported the creation of a pedagogical tool that facilitates reading in French as a second language. The tool was designed to enhance comprehension of links between texts (similarities and differences between four provided texts), comprehension of sources in connection with content (who says what), and detailed comprehension. The results reinforce some theories about reading in a second language and lead to suggestions for teaching.

\section{INDEX}

Mots-clés : lecture en langue seconde, compréhension écrite, lecture multidocumentaire, outil pédagogique

Keywords : second language reading, reading comprehension, multidocumental reading, pedagogical tool

\section{AUTEUR}

\section{GÉRALDINE CHARLES-DOMINIQUE}

Géraldine Charles-Dominique est chercheure associée au laboratoire FORELL (Formes et Représentations en Linguistique et Littérature - EA 3816) de l'Université de Poitiers.

Courriel : geraldine.charles.dominique@univ-poitiers.fr

Toile : http://forell.labo.univ-poitiers.fr/ 
Adresse : Maison des sciences de l'homme et de la société, Bâtiment A5, 5 rue Théodore Lefebvre, TSA 21103, 86073 Poitiers Cedex 9, France. 